\title{
Darstellung der Kindheit während der Kriegsjahre in der deutschsprachigen Kinder- und Jugendliteratur. Ein Beispiel der Veränderung des Genres
}

\section{Jana Baroková}

This article deals with the presentation of the Jewish and non-Jewish childhood during the Second World War in the German written children and juvenile literature. The most important features and differences of both literatures were defined and with help of various examples of the primary literature substantiated. An extra attention was aimed at the Diary of Anne Frank. By the Comic-form of the Diary of Anne Frank I observed the methods and procedures how by the old topics of the juvenile literature to develop them attractive for young readers. Finally I compared the content of Diary of Anne Frank with the Comic-form of the book.

Second World War - children and juvenile literature - Jewish childhood - non-Jewish childhood - Diary of Anne Frank - comic-version of Anne Frank

Dieser Artikel befasst sich mit der Darstellung der jüdischen und nichtjüdischen Kindheit während des Zweiten Weltkrieges in der deutschsprachigen Kinder- und Jugendliteratur. Die wichtigsten Merkmale und Unterschiede von beiden Literaturen wurden definiert und mit Hilfe der ausgewählten Beispiele aus der Primärliteratur konkretisiert. Ein besonderes Augenmerk wurde auf das Anne Frank Tagebuch gerichtet. Bei der Gestaltung des Tagebuches der Anne Frank als Comics war ich bemüht, die Methoden und Vorgehen zu beobachten, wie die alt werdenden Themen der Jugendliteratur den jungen Lesern attraktiv und zugänglich gemacht werden können. Letztendlich habe ich die formale und inhaltliche Seite der ComicFassung mit der Originalfassung des Werkes Anne Frank Tagebuch verglichen und der kritischen Analyse unterworfen.

Zweiter Weltkrieg - deutsche Kinder- und Jugendliteratur - jüdische Kindheit - nichtjüdische Kindheit - Tagebuch der Anne Frank - Anne Frank als Comic

\section{Einführung}

In diesem Artikel werde ich mich mit der Darstellung der Kindheit in der deutschsprachigen Kinder- und Jugendliteratur befassen. Die ganze Problematik wird mit der Perspektive des Abstandes von mehr als einem halben Jahrhundert nach dem Ende des Zweiten Weltkrieges und des Holocausts betrachtet. Ich werde mich zuerst einigen Aspekten widmen, die in ihrer Gesamtheit das Thema „Kriegskindheit bzw. -jugend" betreffen. Da die Familie über Jahrhunderte hinweg ein zentrales Thema der Literatur ist, steht im Mittelpunkt des Interesses der Schriftstellerinnen und Schriftsteller ihre eigene Kindheit, die sie literarisch darstellen wollen (vgl. Wyrobnik 2008: 13-25). Wenn dies nicht der Fall ist, schildern die Autorinnen und Autoren die Kindheit und Erlebnisse ihrer Freunde und ihrer nahe stehenden Menschen wie z. B. Kirsten Boie im Werk Monis Jahr (2003) oder Mirjam Pressler im Roman Malka Mai (2002). Es zeigt sich sowohl in Österreich als auch in 
Deutschland, dass sich viele Autorinnen und Autoren mit ihrer eigenen Kindheit erst dann literarisch befassen, nachdem sie sich mit der Geschichte ihrer Familie auseinandergesetzt haben. So äußert sich z. B. die österreichische Autorin Renate Welsh hinsichtlich ihres Buches Dieda oder das fremde Kind (2002), wo ihre einstige Beziehung als eines quasi nur geduldeten Kindes in der Familie ihrer Stiefmutter während des Zweiten Weltkrieges thematisiert wird, wie folgt:

So lange ich die Geschichte für rein privat hielt und selbst nicht damit im Reinen war, so lange ich noch in einem Rundumschlag mit der Familie meiner Stiefmutter "fertigwerden“ wollte, konnte ich mir nicht erlauben darüber zu schreiben. Inzwischen weiß ich, dass meine Familie auch stellvertretend für viele andere steht, möglicherweise war das Schreiben ein notwendiger Akt der Befreiung. ${ }^{1}$

\section{Aufarbeitung des Themas "Kriegskindheit" für die junge Generation}

Seit dem Ende des Zweiten Weltkrieges fächern sich die Generationen, die Gott sei Dank keinen Krieg mehr erlebt haben, langsam auf. Die Großeltern der heutigen Jugendlichen gehören allmählich der Altersgruppe der Kriegskinder an; die Akteure des Nationalsozialismus sind die bereits verstorbenen Urgroßeltern. Mit wachsendem zeitlichem Abstand von der Zeit des Nationalsozialismus gewinnt die Frage an Bedeutung, auf welche Weise die junge Generation den Zugang zu dieser historischen Epoche finden kann und welche Bedeutung diese Erkenntnis für junge Menschen hat. Unumstritten sind hier solche literarischen Werke von Bedeutung, in denen die Bedeutung der Vergangenheit für die Gegenwart thematisiert wird.

Zur offiziellen Gedenkkultur gehören Gedenkstätten, Museen und Geschichtsvereine. Die Vergangenheit wird in der Literatur und in den Medien aufarbeitet. Einen weiteren Bereich bildet der schulische Geschichts- und Deutschunterricht. Generell wird von einer „Mediatisierung “des Nationalsozialismus und des Holocausts gesprochen (vgl. Ewers und Gremmel 2008: 27-50). Es kommt zu Veränderungen des Genres des jeweiligen Originalwerkes, wie es bei der ComicAufarbeitung des Tagebuches der Anne Frank der Fall ist. Auf dieses Thema werde ich unten ausführlich eingehen.

1 Private Äußerung der Schriftstellerin in der Korrespondenz mit dem Studenten Josef Fiala, Pädagogische Fakultät der Masaryk-Universität, 2005. 


\subsection{Formen der Darstellung der Kriegskindheit in der Kinder- und Jugendliteratur}

Die heutige Literaturforschung beschäftigt sich in wesentlichem Maße mit der Frage des Umgangs mit der Verantwortung für den Krieg und für die Gräuel dieses Krieges (vgl. Shavit 2008: 51-69). Zohar Shavit, Professorin für Literaturwissenschaft an der School of Cultural Studies der Universität Tel Aviv/Israel, spricht von einem überwiegenden Narrativ, d. i. von einer signifikanten Form der realistischen Erzählung im Rahmen der Kinder- und Jugendliteratur: In den meisten Kinder- und Jugendromanen wird die Vorkriegszeit, der Kriegsausbruch bzw. die Nachkriegszeit geschildert. Weniger werden die direkten Kriegsjahre 1941-1945 geschildert. Wenn diese Zeit dennoch thematisiert wird, wie dies z. B. im Roman Lena, unser Dorf und der Krieg (2004) von Käthe Recheis oder im Roman Dieda oder das fremde Kind (2002) von Renate Welsh der Fall ist, bilden die Kriegsereignisse bzw. die Konsequenzen des Krieges für die Zivilbevölkerung wie Hunger, Not, Gefahr, Bedrohung etc. eine ferne Kulisse für die alltäglichen Sorgen der Kinder. Sie dringen nur gefiltert durch die Privatsphäre der Erwachsenen und durch die aufgrund des Krieges veränderte Atmosphäre im Elternhaus in die kindliche Seele ein. In einigen Werken wird die Evakuierung, (ggf. Vertreibung) der Deutschen aus Böhmen und Mähren vom Anfang des Jahres 1945 geschildert. Zu solchen Werken gehören Krücke (1986) und Reise gegen den Wind (2000) von Peter Härtling oder der Roman Überleben! (2005) von Gudrun Pausewang. Beide Autoren beschränken sich bei der Schilderung der Odyssee auf einen eng umrissenen geographischen Bereich, betrachtet aus der eingeschränkten Perspektive der Kinder bzw. Jugendlichen. Im Roman Reise gegen den Wind bildet die kleine Grenzstadt Laa an der Thaya an der österreichisch-mährischen Grenze die verschlafene Kulisse eines großen Abenteuers gesehen mit den Augen des zwölfährigen Protagonisten Bernd. Im Text kommen immer wieder Ereignisse vor, die Bernd als Kind nicht versteht bzw. die außerhalb seiner Vorstellungskraft liegen. Das Kind hat doch kindliche Sorgen und der Krieg soll seinen Platz in der Welt der Erwachsenen haben. Die sog. „heile Welt“ ist in der Episode im Wald zu spüren, wo Bernd kurz vor der Abreise mit seiner Tante aus Laa an der Thaya den geheimnisvollen "fabelhaften Herrn Meier" tot im Gras liegend findet. Die vorherigen dunklen Geschäfte und Machenschaften von Herrn Meier tragen für den Jungen nur unklare und vage Umrisse. Nach Zohar Shavit

[...] entbindet die Kinderperspektive die Autoren von der Pflicht, die historischen Ereignisse vertiefend zu behandeln. Das erzählende Kind weiß wenig, und sein Wissen über die Geschehnisse ist von Naivität und Direktheit geprägt. Zudem ist die Fähigkeit des Kindes darauf beschränkt, Zusammenhänge zwischen einzelnen nicht direkt aufeinander folgenden Ereignissen herzustellen und sie zu verstehen bzw. zu interpretieren. Die Kinderperspektive ermöglicht die fragmentarische und euphemistische Darstellung von Ereignissen und befreit 
den Autor von der Pflicht, Ursache und Wirkung zueinander in Beziehung zu bringen. (Shavit 2008: 57)

Ich schließe mich der Ansicht Zohar Shavit an, dass die Kinder die Wahrheit über die Generation ihrer Eltern und Großeltern erfahren sollten. Ich meine aber dennoch, dass die von mir oben genannten Romane von Peter Härtling und Gudrung Pausewang die Kinder in ihrem Prozess des Begreifens der Welt und der Gegenwartsgeschichte nicht einschränken. Die Kinderbuchautoren haben nicht die Pflicht, die Kriegsereignisse aus dem Blickwinkel des Historikers zu betrachten. Der Krieg bildet in diesen Büchern die schreckliche Alltagskulisse des kindlichen Wahrnehmens und Erlebens der Welt.

\subsection{Darstellung der jüdischen Kindheit und Jugend während des Zweiten Weltkrieges}

Der Begriff „Zweiter Weltkrieg“ bezeichnet die Jahre zwischen 1939 und 1945. Was die jüdische, in Deutschland lebende Bevölkerung angeht, so war diese bereits seit 1933 von Seiten der Nationalsozialisten starken Repressionen und konsequenter Ausgrenzung ausgesetzt. Die Historiker arbeiten seit den 1950er Jahren Themen wie „die Geschichte des Dritten Reiches“, die „Vernichtung der Juden“, die „Endlösung der Judenfrage“ oder „Holocaust“ bzw. „S(c)hoa(h)“ auf. Die deutsche Literaturforscherin Gabriele von Glasenapp befasst sich in ihrem Artikel "Wir haben das nicht unversehrt überstanden" (Glasenapp 2008: 127-149) mit den so genannten jüdischen Kindheitsautobiographien, also mit Texten, in denen die jüdischen VerfasserInnen retrospektiv über ihre Kindheit während des Nationalsozialismus erzählen. Diese Einschränkung begründet die Autorin mit dem folgenden Umstand:

Nur innerhalb des Deutschen Reiches waren jüdische Kinder und Jugendliche von Beginn an, d. h. spätestens seit Ende Januar 1933, den Regime typischen entwürdigenden, diskriminierenden und ausgrenzenden Maßnahmen unterworfen, die in der Retrospektive als Vorgeschichte des Holocaust angesehen werden müssen. (Glasenapp 2008: 129)

Innerhalb der Holocaustliteratur sind autobiographische Texte - die sog. Kindheitsautobiographien - stark vertreten, wobei in der Forschung heute die Meinung überwiegt, dass die einst strenge Grenze zwischen den Begriffen „Autobiographie“ und „Roman“ allmählich verschwindet. Jede Autobiographie enthält nämlich auch fiktionale Elemente. Im Rahmen dieser Kategorie spricht man von einer großen Heterogenität der Texte: In einigen von ihnen wird eine in Deutschland verbrachte, jüdische Kindheit geschildert, wie z. B. im Roman Damals 
war es Friedrich (1961) von Hans Peter Richter, bei anderen handelt es sich um die Darstellung der eigenen Kindheit in der Emigration, wie z. B. im Roman Als Hitler das rosa Kaninchen stahl (1971) von Judith Kerr. Eine wichtige Rolle spielt weiter die Tatsache, dass einige der Originalausgaben der Autobiographien in anderen Sprachen als Deutsch verfasst wurden und erst nachträglich ins Deutsche übersetzt wurden. So wurde z. B. das zuletzt genannte Werk von Judith Kerr im Original auf Englisch unter dem Titel When Hitler Stole Pink Rabbit veröffentlicht.

Je nachdem, was im Werk geschildert wird, unterscheidet Gabriele von Glasenapp fünf Hauptrichtungen der Darstellung der jüdischen Autobiographien in der Kinder- und Jugendliteratur: Erstens handelt es sich um die Situation, wo die Kindheit in Deutschland im Herbst 1938 durch die Abschiebung des Protagonisten und seiner Familie aus Deutschland nach Polen wegen der Absenz deutscher Staatsangehörigkeit beendet wird (wobei dahinter noch andere Gründe versteckt waren). Zweitens handelt es sich um die Emigration der Familie aus Deutschland, entweder 1933 kurz nach der Machtergreifung der Nazis oder erst 1938. Hier ist zu unterscheiden, ob es sich um ein später von den Nazis besetztes Land handelte, wie dies z. B. bei Anne Frank (die Niederlande) der Fall war, oder um ein sicheres Land wie z. B. die USA. Im dritten Fall handelt es sich um Autobiographien, deren jüdische Protagonisten mit Hilfe von Freunden versucht haben, im Untergrund zu überleben. Auf diese Weise greift der Krieg z. B. in das Leben der kindlichen Protagonistin im Jugendroman Malka Mai (2001) von Mirjam Pressler ein. Die vierte Kategorie betrifft solche Kinder und Jugendliche, die nur einen jüdischen Elternteil hatten. Oft ließen ihre Eltern diese Kinder bewusst im Unklaren, was später zu schweren Krisen der Protagonisten führte. Die letzte Gruppe von Texten bilden nach Gabriele von Glasenapp jene Autobiografien, deren Protagonisten in ein KZ deportiert wurden. Trotz der oben angeführten Differenzierung sind diese Autobiografien doch auf einen gemeinsamen Nenner zu bringen: Alle sind nämlich als ein Dokument der Erinnerung gedacht.

\subsection{Einige Motive der jüdischen Kindheit am Beispiel des Tagebuches der Anne Frank}

Das Tagebuch der Anne Frank sind literarisch verfasste Tagebuchaufzeichnungen von Anne Frank (1929-1945), die 1947 als Het Achterhuis niederländisch (dt. „Das Hinterhaus"), 1950 dann deutsch als Das Tagebuch der Anne Frank veröffentlicht wurden. Die letzte deutsche Übersetzung stammt von Mirjam Pressler.

Bei der Darstellung des jüdischen Familienlebens bedeutet die Familie für die ProtagonistInnen primär einen ruhigen Hafen voll von emotionaler Sicherheit, wo jüdische Traditionen aufrechterhalten werden. In dem bereits aus dem Amsterdamer Versteck geschriebenen Brief an ihre fiktive Freundin Kitty von Montag, dem 7. Dezember 1942, schildert Anne den Verlauf der mit dem Ende des Jahres 
verbundenen jüdischen Feste. Sie schreibt über Chanukka, was auf Deutsch „Weihe“ bedeutet. Es handelt sich um das jüdische Fest der Tempelweihe im Dezember:

Chanukka und Nikolaus fielen dieses Jahr fast zusammen, der Unterschied war nur ein Tag. Für Chanukka haben wir nicht viele Umstände gemacht, ein paar hübsche Sächelchen hin und her und dann die Kerzen. Da ein Mangel an Kerzen herrscht, wurden sie nur zehn Minuten angezündet, aber wenn das Lied nicht fehlt, ist das auch ganz gut. Herr van Daan hatte einen Leuchter aus Holz gemacht, sodass das auch geregelt ist. (Frank und Pressler 1991: 82)

Je länger der Alltag der ins Absurde getriebenen Lebenssituationen, voll von Gefahr und Lebensbedrohung, dauerte, wie es bei der Familie Frank nach anderthalb im Versteck verbrachten Jahren der Fall war, desto mehr beginnt Anne mit der Zeit die anderen Untergetauchten, wie ihre Eltern und ihre Schwester Margot oder auch das Ehepaar van Daan und den Zahnarzt Albert Dussel mit einem kritischen Abstand und der beharrlichen Widerspenstigkeit wahrzunehmen. Die verbalisierten Gefühle gelten als Ventil für das Mädchen infolge der für alle Beteiligten bis aufs Äußerste zugespitzten, bedrohlichen Lage. Anne ahnt, dass die festen Wurzeln aufgrund der äußeren Umstände zerbrochen, die Eltern dagegen ohnmächtig sind und ihr keinen Schutz mehr gegenüber den Gefahren der äußeren Welt bieten können. Der Brief an Kitty von Samstag, dem 30. Januar 1943, lässt den Gefühlen des seelischen Schmerzens und des Selbstmitleids freien Lauf:

Ich dampfe vor Wut und darf es nicht zeigen. Ich würde am liebsten mit den Füßen aufstampfen, schreien, Mutter gründlich durchschütteln, weinen und was weiß ich noch alles wegen der bösen Worte, der spöttischen Blicke, der Beschuldigungen, die mich jeden Tag aufs Neue treffen wie Pfeile von einem straff gespannten Bogen und die so schwer aus meinem Körper zu ziehen sind. Ich möchte Mutter, Margot, van Daan, Dussel und auch Vater anschreien: Lasst mich in Ruhe! Lasst mich endlich mal eine Nacht schlafen, ohne dass mein Kissen nass von Tränen ist, meine Augen brennen und Schmerzen in meinem Kopf hämmern. (Frank und Pressler 1991: 89)

Neben der Familie bedeutet für die Kinder die Schule einen wichtigen Ort ihrer Entwicklung und Anpassung an die Gesellschaft. Vor 1933 besuchten auch jüdische Kinder vorwiegend allgemeine Schulen. Zuerst war die Schwester von Anne Frank, Margot, Schülerin an einer eher traditionellen Schule in Amsterdam, Jekerstraat, Anne ging am Anfang in einen Kindergarten, der einer MontessoriSchule angeschlossen war. Infolge der gespannten politischen Atmosphäre mussten beide Schwestern ihr übliches schulisches Umfeld verlassen und gingen ins Jüdische Lyzeum. Anne schildert diese Veränderung in ihrem Leben wie folgt: 
Ich ging bald in den Kindergarten der Montessorischule. Dort blieb ich bis sechs, dann kam ich in die erste Klasse. In der 6. Klasse kam ich zu Frau Kuperus, der Direktorin. Am Ende des Schuljahres nahmen wir einen herzergreifenden Abschied voneinander und weinten beide, denn ich wurde am Jüdischen Lyzeum angenommen, in das Margot auch ging. (Frank und Pressler 1991: 20)

\subsection{Die Darstellung des Lebens Annes Frank auf dem Weg von der Gattung Tagebuch zur Gattung Comics}

Abschließend werde ich mich den veränderten künstlerischen Mitteln innerhalb der Genres widmen, die für die retrospektive Auseinandersetzung mit der Zeitgeschichte eingesetzt werden. Das Tagebuch der Anne Frank wurde seit seiner Veröffentlichung bereits als Theaterstück, und zwar in der Bearbeitung von Frances Goodrich und Albert Hackett unter dem Titel The Diary of Anne Frank 1955 am Broadway in New York aufgeführt. In demselben Jahr gewann diese Dramatisierung den Pulitzer-Preis in der Sparte Drama. (Vgl. Fischer 2008: 163).

Ich möchte mich nun noch kurz mit der Darstellung unseres Themas als Comic beschäftigen. In diesem konkreten Fall handelt es sich um die Bearbeitung des Tagebuches von Anne Frank als Comic-Geschichte mit dem Titel Das Leben von Anne Frank. Eine grafische Biografie (2010) von den Autoren Sid Jacobson und Ernie Colón. Im Zeitalter der neuen digitalen Medien und des Internets wächst auch die Bemühung der Autoren, neue potentielle Adressaten-Leser zu gewinnen. Immer mehr Aufmerksamkeit wird der Aufgabe gewidmet, zeitgeschichtliche Themen neu aufzuarbeiten. In der Comic-Gestaltung des Themas „Anne Frank“ handelt es sich nicht nur um die Bearbeitung des Tagebuches von Anne Frank, sondern um eine ausführliche Biografie von Anne, ihren Eltern und ihrer um drei Jahre älteren Schwester Margot. Den umfangreichen geschichtlich-sozialen Hintergrund der Biografie bilden neun Kapitel mit den Titeln „Die Nazis auf dem Vormarsch“, „Amsterdam“, „Unter deutscher Besatzung“, „Die acht Untertaucher“, „Das neue Jahr“, „Entdeckt“, „Die Geschichte geht weiter“, wobei „Das Tagebuch“ selbst die zentrale Stelle des Werkes einnimmt. Wegen der besseren Übersicht ist das Buch um eine Zeittafel und um Tipps zum Weiterlesen ergänzt.

Bei dem vorliegenden Werk handelt es sich um eine konventionelle dialogisierte Comic-Geschichte, in der die klassischen Techniken des Comic-Genres angewendet werden. Die Protagonisten äußern sich in ihren Aussagen mit Hilfe von Sprechblasen, ihre Gedanken, Wünsche bzw. Gefühle und nicht verbalisierte Situationen werden dann mit Hilfe von Denkblasen in Form von schwebenden wolkenartigen Gebilden vermittelt. Einleitende Sätze sowie Kommentare zur politischen Situation oder zeitlichen Zäsuren bzw. Schauplatzwechsel markierende Texte treten in Textrahmen am Rande der Bilder auf (vgl. Weinkauff-Dolle 2008: 118). Ein fester, schwarzer Rahmen um die Kommentare herum deutet visuell an, dass über Geschehnisse 
der Außenwelt berichtet wird, von der die acht untergetauchten Menschen völlig abgeschnitten sind. Außerdem werden in solchen Kommentaren zum Vergleich auch die entsprechenden Originaltexte aus Annes „Tagebuch“ angeführt. So schreibt Anne im Brief an Kitty vom 9. Oktober 1942:

Liebe Kitty! Nichts als traurige und deprimierende Nachrichten habe ich heute. Unsere jüdischen Bekannten werden gleich gruppenweise festgenommen. Die Gestapo geht nicht im Geringsten zart mit diesen Menschen um. Sie werden in Viehwagen nach Westerbork gebracht, dem großen Lager in Drente. Miep hat von jemandem erzählt, der aus Westerbork geflohen ist. Es muss dort schrecklich sein. (Frank und Pressler 1991: 64)

Die entsprechende Tafel in der Comic-Fassung enthält den folgenden Text:

Die Nachrichten aus der Außenwelt wurden immer schlimmer ... Am 9. Oktober schrieb Anne: „Unsere jüdischen Bekannten werden gleich gruppenweise festgenommen“, und ,in Viehwagen nach Westerbork gebracht“. (Jacobson und Colón 2010: 89)

Auf derselben Tafel schwebt über einem Radiogerät der nächste Kommentar, der über die aktuellen Ereignisse in der Außenwelt informiert: ,Juden werden planmäßig mit Maschinengewehren, Handgranaten und sogar Giftgas getötet" (Jacobson und Colón 2010, 89). Der Text ist kursiv verfasst und ist angebracht im festen Rahmen, der mit einem blitzförmigen spitzigen Pfeil am Ende ausgestattet ist. Dieses visuelle Zeichen ist für die dringende Gefahr und Bedrohung der handelnden Personen bestimmend und hat einen direkten Bezug zum Kontext. Im Brief an Kitty vom 9. Oktober aus dem Tagebuch kommt diese Passage nicht vor. Die blitzförmige Endung des Rahmens im Comic verschärft das Wahrnehmen der bedrohlichen Lebenssituation der Protagonisten, die darüber hinaus durch den besorgten Gesichtsausdruck aller im Bildkasten versammelten Untergetauchten unterstrichen ist.

In der Fassung der Autoren Jacobson und Colón wird die innere Entwicklung Annes folgend dargestellt: Dort, wo längere Passagen vorkommen (z. B. die Stelle, an der sich Anne in ihrem Brief mit ihrer emotionalen Reifung Kitty anvertraut), sind sie bemüht, die signifikantesten und aussagekräftigsten Ausschnitte aus dem Originaltext im Comic-Rahmen zu platzieren. So findet man z. B. im Tagebuch die folgende Aussage:

Peter ist lieb und gut, trotzdem, ich darf es nicht leugnen, enttäuscht mich vieles. Vor allem seine Abkehr von der Religion, seine Gespräche über Essen und noch andere so widersprüchliche Dinge gefallen mir nicht. Trotzdem bin ich fest davon überzeugt, dass wir nach unserer ehrlichen Abmachung nie Streit 
bekommen werden. Peter ist friedliebend, verträglich und sehr nachgiebig. Er lässt sich von mir viel mehr sagen, als er seiner Mutter zugesteht. Er versucht mit viel Hartnäckigkeit, die Tintenflecke aus seinen Büchern zu entfernen und Ordnung in seinen Sachen zu halten. Aber warum bleibt sein Inneres dann innen, und ich darf nie daran rühren? (Frank und Pressler 1991: 296)

In der entsprechenden Tafel der Comic-Fassung steht der folgende Text: „An Peter enttäuscht mich vieles ... Warum bleibt sein Innerstes innen, und ich darf nie daran rühren? ... Ich vermisse das Echte und weiß doch sicher, dass es da ist!" (Jacobson und Colón 2010, 89).

Die Passage des Tagebuches, in der sich Anne ihrer Schwester Margot anvertraut, dass sie die Lehrerin Frau Kuperus vermissen würde, ist in der Comic-Fassung ziemlich unterschiedlich dargestellt. Frau Kuperus spricht Anne vor den anderen Kindern nur ganz kurz an: „Tut mir so leid, Anne“. Diese Konstatierung ist in einer Sprechblase angebracht, wobei darunter noch eine Denkblase mit dem Text folgt: „Mir war gar nicht klar, wie viele jüdische Schüler ich habe" (Jacobson und Colón 2010: 63).

Mit Billigung der Nationalsozialisten gelang es den jüdischen Gemeinden, für jüdische Kinder Schulen und Institutionen zu schaffen, in denen sie ihre Identität positiv wahrnehmen und sich wohl fühlen konnten. In der Comic-Fassung findet man zwei Bilder über den Alltag im jüdischen Lyzeum, in das Anne und Margot gingen, mit dem folgenden Text:

Für die beiden Mädchen stellte sich die jüdische Schule jedoch als positiv heraus. Die Lehrer arbeiteten eng mit den Kindern zusammen und schufen so ein Gemeinschaftsgefühl. [...] Denen, die den Holocaust überlebten, sollte es als seine glückliche Zeit in Erinnerung bleiben. (Jacobson und Colón 2010: 63)

Beide Mädchen besuchten Jugendvereine, in denen ihnen in Form von verschiedenen Freizeitbetätigungen nützliche Informationen über Geschichte und Kultur der Juden beigebracht wurden. Bei Jacobson und Colón heißt es dazu: „Zu dieser Zeit begannen Anne und Margot Lektionen über das Judentum bei Rabbi Mehler zu nehmen. [...] und Margot trat außerdem einer zionistischen Jugendgruppe bei“ (Jacobson und Colón 2010: 62). Beide Texte sind in einem einfachen Rahmen als eine objektive Konstatierung angebracht.

Diesem Kontext entspricht nur in marginalen Umrissen die Passage im Tagebuch über Annes Beziehung zu Zionismus: 
Mittwochs abends habe ich immer frei, weil meine Großeltern glauben, ich gehe zu Schnitzen, aber ich gehe zum Treffen der Zionistischen Partei. Das darf ich eigentlich nicht, weil meine Großeltern sehr gegen den Zionismus sind. Ich bin zwar auch nicht fanatisch, aber ich interessiere mich dafür. (Frank und Pressler 1991: 28)

Unterschiedlich gegenüber den Aufzeichnungen im Tagebuch ist in der ComicFassung Annes Wahl der fiktiven Freundin Kitty, Empfängerin ihrer Briefe, dargestellt. Im Tagebuch empfindet die Protagonistin schmerzhaft die Tiefe ihrer Gedankenwelt, in die sie ihre realen Freundinnen nie einweihen könnte:

Ich kann mit keinen von meinen Bekannten etwas anderes tun als Spaß machen, ich kann nur über alltägliche Dinge sprechen und werde nie intimer mit ihnen. Das ist der Haken. Vielleicht liegt dieser Mangel an Vertraulichkeit auch an mir. Jedenfalls ist es so, leider, und nicht zu ändern. [...] Um nun die Vorstellung der ersehnten Freundin in meiner Phantasie noch zu steigern, will ich nicht einfach Tatsachen in mein Tagebuch schreiben wie alle andern, ich will dieses Tagebuch die Freundin selbst lassen, und diese Freundin heißt Kitty. (Frank und Pressler 1991: 18-20)

In einem fest umrahmten Text der Comic-Fassung findet der Leser die vereinfachende Konstatierung des auktorialen Erzählers über Annes Sehnsucht nach Freundinnen, jedoch nichts von ihren Hemmungen gegenüber ihnen:

Ich Hinterhaus vermisste Anne ihre Freundinnen. Angeregt durch ein Buch begann sie, ihre Einträge in Form von Briefen an imaginäre Freundinnen (Kitty, Emmy, Jet, Pop, Marianne, Conny, Phien, Lou) und an eine reale, Jacquueline, zu schreiben. (Jacobson und Colón 2010: 88).

In der Denkblase findet man nur den folgenden Text: „Liebe Kitty, gestern habe ich an Emmy und Jettje geschrieben, aber am liebsten schreibe ich dir ..." (Jacobson und Colón 2010: 88).

In der Fassung des Tagebuches von Mirjam Pressler gibt es dagegen nur an Kitty adressierte Briefe. Die häufigste Anrede ist „Liebe Kitty“, selten kommen die Anreden „Beste Kitty“, „Liebste Kitty!“, „Liebste Kit!“, in einem Fall ist die Anrede vollkommen unterschiedlich, und zwar „Allerliebster Schatz!“, die man auf Seite 213 findet. Genauso variiert auch die Unterschrift der Protagonistin. Am häufigsten erscheint die Unterschrift „Deine Anne“, oder einfach nur „Anne“, ab und zu jedoch auch z. B. „Anne Frank“, oder „Deine Anne M. Frank“. Ein Brief ist sogar mit der Unterschrift „Deine ergebene Anne M. Frank“ versehen. 
Abschließend kann konstatiert werden, dass die Comic-Gestaltung des Tagebuches der Anne Frank zwar alle narrativen Schwerpunkte des Originalwerkes enthält, die Technik der Darstellung entspricht jedoch den elementaren Grundsätzen des Genres Comics. Die Comic-Gestaltung des Themas erfüllt meiner Meinung nach die Funktion der Verschiebung des behandelten Stoffes in das Zeitalter der modernen Medien, die mit der Geradlinigkeit und Flächenhaftigkeit der Aussage und der für das Genre typischen sporadischen Ausdrucksweise auf den Rezipienten unnötig anschaulich und vereinfachend wirken.

\section{Schlusswort}

Im vorliegenden Artikel habe ich mich mit der Darstellung der Kindheit in der Kinder- und Jugendliteratur befasst, deren Hauptmotiv das Erleben der Gegenwartsgeschichte, und zwar des Zweiten Weltkrieges ist. Beim Tagebuch der Anne Frank und dessen Gestaltung als Comic war ich bemüht, die Methoden und das Vorgehen zu zeigen, wie die klassisch werdenden Themen der Kinderund Jugendliteratur auch für junge Leser attraktiv und zugänglich aufbereitet werden können. Schließlich habe ich die formale und inhaltliche Seite der ComicFassung mit der Originalfassung des Werkes Anne Frank Tagebuch verglichen und kommentiert.

\section{Primärliteratur}

Boie, Kirsten (2003): Monis Jahr. Hamburg, Verlag Friedrich Oetinger.

Frank, Otto / Pressler, Mirjam (1991): Anne Frank Tagebuch. Frankfurt am Main, S. Fischer Verlag.

Härtling, Peter (2000): Reise gegen den Wind. Weinheim Basel, Beltz und Gelberg Verlag. Jacobson, Sid / Colón, Ernie (2010): Das Leben von Anne Frank. Eine grafische Biografie. Hamburg, Carlsen Verlag.

Kerr, Judith (1971): Als Hitler das rosa Kaninchen stahl. A. d. Engl. von Annemarie Böll. Ravensburg, Maier 1973.

Pausewang, Gudrun (2005): Überleben! Ravensburg, Ravensburger Buchverlag Otto Meier. Pressler, Mirjam (2001): Malka Mai. Weinheim, Beltz und Gelberg Verlag.

Richter, Hans Peter (1980): Damals war es Friedrich. München, Deutscher Taschenbuch Verlag.

\section{Sekundärliteratur}

Dolle-Weinkauff, Bernd (2008): Kindheitsgeschichten aus dem Krieg als Cyberfiktion, in: Glasenapp von, Gabriele / Ewers, Hans-Heino (Hg.): Kriegs- und Nachkriegskindheiten. Studien zur literarischen Erinnerungskultur für junge Leser. Frankfurt am Main, Peter Lang Verlag. 
Ewers, Hans-Heino/Gremmel, Caroline (2008): Zeitgeschichte, Familiengeschichte und Generationenwechsel. Deutsche zeitgeschichtliche Jugendliteratur der 1990er und 2000er Jahre im erinnerungskulturellen Kontext, in: Glasenapp von, Gabriele / Ewers, Hans-Heino (Hg.): Kriegs- und Nachkriegskindheiten. Studien zur literarischen Erinnerungskultur für junge Leser. Frankfurt am Main, Peter Lang Verlag.

Fischer, Gerhard (2008): Anne Frank, Naomi Kirschbaum und Inge Deutschkron auf der Bühne: Über die Dramatisierungen jüdischer Mädchenschicksale zur Zeit des Nationalsozialismus und des Holocaust, in: Glasenapp von, Gabriele / Ewers, Hans-Heino (Hrsg.): Kriegs- und Nachkriegskindheiten. Studien zur literarischen Erinnerungskultur für junge Leser. Frankfurt am Main, Peter Lang Verlag.

Glasenapp von, Gabriele (2008): „Wir haben das nicht unversehrt überstanden“. Jüdische Kindheit und Jugend während des Dritten Reiches im Spiegel autobiographischer Texte, in: Glasenapp von, Gabriele / Ewers, Hans-Heino (Hrsg.): Kriegs- und Nachkriegskindheiten. Studien zur literarischen Erinnerungskultur für junge Leser. Frankfurt am Main, Peter Lang Verlag.

Shavit, Zohar (2008): Es war einmal ein Krieg. Jüdische und nichtjüdische Kriegs- und Nachkriegskindheit und -jugend in der Kinder- und Jugendliteratur, in: Glasenapp von, Gabriele / Ewers, Hans-Heino (Hrsg.): Kriegs- und Nachkriegskindheiten. Studien zur literarischen Erinnerungskultur für junge Leser. Frankfurt am Main, Peter Lang Verlag.

Wyrobnik, Irit (2008): Familie und NS-Zeit. Erinnerungskulturen zu Beginn der 2000er Jahre, in: Glasenapp von, Gabriele / Ewers, Hans-Heino (Hrsg.): Kriegsund Nachkriegskindheiten. Studien zur literarischen Erinnerungskultur für junge Leser. Frankfurt am Main, Peter Lang Verlag. 